\title{
Role of Reticulocyte Parameters in Anemia of First Trimester Pregnancy: A Single Center Observational Study
}

\author{
Utpal Kumar ${ }^{1}$ Harish Chandra ${ }^{1}$ Arvind Kumar Gupta ${ }^{1}$ Neha Singh ${ }^{1}$ Jaya Chaturvedi \\ ${ }^{1}$ Department of Pathology and Laboratory Medicine, All India \\ Institute of Medical Sciences, Rishikesh, Uttarakhand, India \\ 2Department of Obstetrics and Gynecology, All India Institute of \\ Medical Sciences, Rishikesh, Uttarakhand, India

\begin{abstract}
Address for correspondence Harish Chandra, MD, Department of Pathology and Laboratory Medicine, All India Institute of Medical Sciences, Rishikesh 249201, Uttarakhand, India
\end{abstract} \\ (e-mail: drharishbudakoti31@yahoo.co.in).
}

J Lab Physicians:2020;12:15-19

\begin{abstract}
Keywords

- pregnancy

- anemia

- reticulocyte

hemoglobin content

- immature reticulocyte fraction

Introduction The diagnosis of anemia in pregnancy should be early to prevent complications to mother and baby. It should be simple and safe, and therefore feasibility of reticulocyte parameters may prove beneficial in this regard. Newer reticulocyte parameters have not been studied extensively especially in pregnancy. The present study was conducted to evaluate reticulocyte hemoglobin content (Ret-He), immature reticulocyte fraction, and reticulocyte count and to compare them with serum ferritin, mean corpuscular volume (MCV), and mean corpuscular hemoglobin $(\mathrm{MCH})$ in assessment anemia in pregnancy.

Material and Methods Prospective study included first trimester pregnant females where hemoglobin, MCV, and $\mathrm{MCH}$ were categorized and statistically analyzed with serum ferritin and reticulocyte parameters.

Results The study included 155 first trimester pregnant females showing statistical difference in Ret-He between all categories of hemoglobin and MCV $(p<0.05)$. ROC curve analysis using $\mathrm{MCV}<83 \mathrm{fl}$ and $\mathrm{MCH}<27 \mathrm{pg}$ showed that area under curve for Ret-He at cutoff of $27.8 \mathrm{ng} / \mathrm{mL}$ was 0.93 (95\% confidence interval $0.90-0.98$, sensitivity $93 \%$, specificity $83 \%$ ). The cutoff of $27.2 \mathrm{ng} / \mathrm{mL}$ Ret-He for the diagnosis of iron deficiency anemia has specificity of $86 \%$, sensitivity $71 \%$, positive predictive value 0.39 , and likelihood ratio of 5.1 .

Conclusion Ret-He is a feasible and easily available parameter which may be helpful in differentiating between microcytic hypochromic and normocytic normochromic anemia along with frank and borderline anemia in first trimester pregnant females. This in turn is essential to initiate an early therapy so as to prevent the maternal and fetal complications.
\end{abstract}

\section{Introduction}

Pregnancy has always been a puzzle for both pathologists and clinicians in interpreting any hematological or biochemical parameters due to marked physiological and associated plasma volume changes. According to World Health Organization (WHO) $40.1 \%$ of total pregnant females are anemic worldwide, and the burden lies mostly on developing
DOI https://doi.org/

$10.1055 / \mathrm{s}-0040-1713585$

ISSN 0974-2727 . and under developed countries. ${ }^{1}$ Out of these, approximately $50 \%$ of anemia is due to iron deficiency with Southeast Asian and African countries contributing about half of global maternal deaths. Therefore, it is essential to diagnose iron deficiency anemia (IDA) as early as possible in pregnancy so as to prevent complications to both mother and the baby. It is also important that the required investigations should not only be simple, safe, feasible but also cost effective for early

(C)2020 by The Indian Association of Laboratory Physicians
License terms

(요 (1) $\Theta \circledast$ 
diagnosis of IDA. Although the gold standard for diagnosing IDA is to evaluate bone marrow iron stores and serum ferritin but it has its own limitations in pregnancy. Bone marrow iron stores should not usually be evaluated in pregnancy because it is an invasive procedure while serum ferritin shows variation in thresholds depending on various physiological changes in pregnancy. ${ }^{2,3}$ Still, serum ferritin has been used routinely and threshold level of less than $15 \mathrm{ng} / \mathrm{mL}$ is usually used to define IDA in pregnancy. ${ }^{4,5}$ Recently, different reticulocyte parameters are being studied in the diagnosis of IDA in various age groups but their analysis exclusively in pregnancy is rarely reported. ${ }^{6,7}$ The major advantage of these reticulocyte parameters is that it can be evaluated easily with recent automated hematology analyzers using routine blood samples. The feasibility and the ease of use of reticulocyte parameters may also prove beneficial in evaluation of IDA in pregnancy. The present study was therefore conducted to evaluate reticulocyte parameters including reticulocyte hemoglobin content (Ret-He), immature reticulocyte fraction (IRF), and reticulocyte count (RC) in diagnosing IDA in pregnancy. In addition, it was also intended to compare these parameters with serum ferritin, mean corpuscular volume (MCV), and mean corpuscular hemoglobin (MCH) in assessment of IDA in pregnant females.

\section{Material and Methods}

Prospective study was undertaken in the hematology section of the institute which included first trimester pregnant females who came for routine obstetrical examination followed by laboratory investigations. The blood samples were collected in ethylene diamine tetra-acetic acid (EDTA) anticoagulant tubes and plain vials after written informed consent. Complete clinical details were taken from all the females at the time of phlebotomy. The EDTA sample was subjected for complete blood count analysis, using Sysmex-XN 1000 Automatic Full Digital Cell Counter (Sysmex Corporation). Hemoglobin level (Hb), MCV, MCH, RC, IRF, and Ret-He that were available on automated analyzer were recorded for every patient. The serum was used for estimation of serum ferritin using ADVIA Centaur XP semi-automated analyzer (Siemens Healthineers) (immunoassay via chemiluminescence). All the samples were subjected to cell counter within 6 hours of collection, but not at a fixed time. Cases that were on hematinic were excluded from the study. According to WHO, females having hemoglobin less than $11 \mathrm{~g} / \mathrm{dL}$ were considered anemic and according to United Kingdom guidelines on the management of iron deficiency in pregnancy threshold of serum ferritin less than $15 \mathrm{ng} / \mathrm{mL}$ was considered significant for IDA. ${ }^{4,8}$ All the data were entered in a spreadsheet and statistical analysis was done using $\mathrm{R}$ software. The comparison of the measured reticulocyte parameters was accomplished by boxplots and Kruskal-Wallis test (with post-hoc Mann-Whitney U test). $p$-Value less than 0.05 was considered statistically significant. The study was approved by Institutional Research and Ethics Committee.

\section{Results}

The study included total 155 first trimester pregnant females with mean age group of 26.7 years. All the females were categorized according to $\mathrm{Hb}, \mathrm{MCV}$, and $\mathrm{MCH}$ in 3, 4 , and 2 categories, respectively as shown in -Table $\mathbf{1}$. It shows that females with $\mathrm{Hb}$ less than $11 \mathrm{~g} / \mathrm{dL}$ were 37 , MCV less than $80 \mathrm{fl}$ were 22, and MCH less than $27 \mathrm{pg}$ were 46. It was observed that median serum ferritin level was $18 \mathrm{ng} / \mathrm{mL}$ and 64 females had serum ferritin level less than $15 \mathrm{ng} / \mathrm{mL}$. - Table 2 shows reticulocyte parameters and serum ferritin levels according to different $\mathrm{Hb}$ categories. It was observed that there was statistical difference in Ret-He between all the categories of $\mathrm{Hb}(p<0.05)$ while IRF was statistically different between categories 2 and 1 and category 3 and $1(p<0.05)$. Serum ferritin was statistically higher in category 3 of $\mathrm{Hb}$ in comparison to category 1 and $2(p<0.05)$. - Table 3 shows reticulocyte parameters and serum ferritin levels according to different MCV categories. It was observed that there was statistically significant difference in Ret-He between all the categories of MCV $(p<0.05)$ except between category 3 and 4. IRF did not show any statistical difference between the different MCV

Table 1 Categorical classification of hematological parameters

\begin{tabular}{|l|l|l|l|l|}
\hline Parameters & Category I & Category II & Category III & Category IV \\
\hline Hemoglobin (gm/dL) & $\begin{array}{l}\text { Anemia }(0-11) \\
\text { Number of cases: } 37\end{array}$ & $\begin{array}{l}\text { Borderline anemia } \\
(11.01-12) \\
\text { Number of cases: } 48\end{array}$ & $\begin{array}{l}\text { Normal }(>12.01) \\
\text { Number of cases: } 70\end{array}$ & \\
\hline $\begin{array}{l}\text { Mean corpuscular } \\
\text { Volume (f) }\end{array}$ & $\begin{array}{l}\text { Severe microcytosis } \\
(0-70) \\
\text { Number of cases: } 7\end{array}$ & $\begin{array}{l}\text { Mild to moderate micro- } \\
\text { cytosis (70.01-80) } \\
\text { Number of cases: } 15\end{array}$ & $\begin{array}{l}\text { Normocytosis } \\
(80.01-100) \\
\text { Number of cases: } 130\end{array}$ & $\begin{array}{l}\text { Macrocytosis }(>100.01) \\
\text { Number of cases: } 3\end{array}$ \\
\hline $\begin{array}{l}\text { Mean corpuscular } \\
\text { hemoglobin }(\%)\end{array}$ & $\begin{array}{l}\text { Hypochromic }(0-27) \\
\text { Number of cases: } 46\end{array}$ & $\begin{array}{l}\text { Normochromic }(>27.01) \\
\text { Number of cases: } 109\end{array}$ & & \\
\hline
\end{tabular}


Table 2 Serum ferritin and reticulocyte parameters in different categories of hemoglobin

\begin{tabular}{|l|l|l|l|}
\hline Parameters (Median value) & Category I & Category II & Category III \\
\hline Serum ferritin (ng/mL) & 10.7 & 13.8 & 23.5 \\
\hline Ret-He (ng/mL) & 28.6 & 30.3 & 31.7 \\
\hline IRF & 13.5 & 11.2 & 9.5 \\
\hline RC & 0.06 & 0.07 & 0.06 \\
\hline
\end{tabular}

Abbreviations: IRF, immature reticulocyte fraction; RC, reticulocyte fraction; Ret-He, reticulocyte hemoglobin.

Table 3 Serum ferritin and reticulocyte parameters in different categories of mean corpuscular volume

\begin{tabular}{|l|l|l|l|l|}
\hline $\begin{array}{l}\text { Parameters } \\
\text { (Median value) }\end{array}$ & Category I & Category II & Category III & Category IV \\
\hline Serum ferritin (ng/mL) & 7.8 & 8 & 20.3 & 62 \\
\hline Ret-He $(\mathrm{ng} / \mathrm{mL})$ & 21.1 & 25.8 & 31.2 & 31.7 \\
\hline IRF & 17.7 & 12.2 & 10.2 & 13.7 \\
\hline RC & 0.07 & 0.06 & 0.06 & 0.08 \\
\hline
\end{tabular}

Abbreviations: IRF, immature reticulocyte fraction; RC, reticulocyte fraction; Ret-He, reticulocyte hemoglobin.

categories. - Fig. 1 shows the boxplot analysis of Ret-He in different categories of $\mathrm{Hb}, \mathrm{MCV}$, and $\mathrm{MCH}$. - Table 4 shows reticulocyte parameters and serum ferritin levels according to different MCH categories. It shows that Ret-He, IRF, and serum ferritin are statistically significant in differentiating hypochromic and normochromic category. It was also observed that RC was not statistically significantly different in any of the category of all analyzed hematological parameters. The analysis of ROC curve using MCV $<83 \mathrm{fl}$ and $\mathrm{MCH}<27 \mathrm{pg}$ shows that area under curve for Ret-He at cut-off of $27.8 \mathrm{ng} / \mathrm{mL}$ is 0.93 (95\% confidence interval [CI] $0.90-0.98$, sensitivity $93 \%$, specificity $83 \%$ ) and for serum ferritin at cutoff of $11 \mathrm{ng} / \mathrm{mL}$ is $0.76(95 \% \mathrm{Cl} 0.66-0.87$, sensitivity $76 \%$, specificity 69\%) (-Fig. 2). The cutoff of $29.4 \mathrm{ng}$ Ret-He for diagnosis of IDA (serum ferritin $<15 \mathrm{ng} / \mathrm{mL}$ ) has specificity of $87 \%$, sensitivity $52 \%$, positive predictive value 0.73 , negative predictive value 0.72 , and likelihood ratio of 3.9. The cutoff of $27.2 \mathrm{ng} / \mathrm{mL}$ of Ret-He for the diagnosis of IDA (serum ferritin $<15 \mathrm{ng} / \mathrm{mL}$ and $\mathrm{Hb}<11 \mathrm{~g} / \mathrm{dL}$ ) as determined by ROC curve had specificity of $86 \%$ and sensitivity of $71 \%$. Further analysis yielded positive predictive value 0.39 , negative predictive value 0.96 , and likelihood ratio of 5.1 for diagnosing IDA.

\section{Discussion}

Ret-He is the measure of the functional iron which is available in hemoglobin of reticulocytes for new red blood cell production while IRF is the proportion of young reticulocytes with the highest RNA content. ${ }^{8}$ These reticulocyte parameters have not only been studied in IDA but also in response to therapy, hereditary spherocytosis, and pyruvate kinase deficiency anemia screening. ${ }^{9,10}$ Serum ferritin, an important parameter has a varied cutoff for diagnosing
IDA in pregnancy ranging from 12 to $30 \mathrm{ng} / \mathrm{mL}^{5,11}$ This variation in serum ferritin in pregnancy may be attributed to different physiological changes occurring in pregnancy that may include second trimester plasma volume expansion, reactive acute phase proteins rise, or third trimester changes in inflammatory measures. ${ }^{2,3,12}$ Therefore the evaluation of easily available reticulocyte parameters may be helpful in diagnosing IDA of pregnancy. It was observed in the present study that Ret-He and IRF were able to differentiate between categories of normal, borderline, or anemic pregnant females according to $\mathrm{Hb}$ while serum ferritin observed no statistical difference between borderline (category II) and anemic (category I) patients. The differentiation between frank anemia and borderline anemia in pregnancy is essential to initiate an early therapy to prevent the maternal and fetal complications and Ret-He and IRF may be helpful in this regard. Previously, it was reported that Ret-He content is an early and accurate predictor of hematological response to oral iron therapy in children. ${ }^{13}$ Few recent studies have also evaluated Ret-He content to assess latent iron deficiency and microcytic red blood cells in adult populations. ${ }^{14,15}$ Chaipokam et al concluded that Ret-He content combined with reticulocyte and blood count is useful in evaluating microcytic red blood cells in Thai patients while Tiwari et al observed that Ret-He may be used in routine screening of latent iron deficiency in blood donors. ${ }^{14,15}$ Although evaluation of Ret-He in pregnant females has been rarely studied but it is observed in the present study that Ret-He and IRF is able to statistically differentiate between normochromic and hypochromic blood picture ( - Table 4 ) while Ret-He was also able to differentiate between severely microcytic, microcytic, and normocytic red blood cells. This observation further suggests that Ret-He may be helpful to differentiate between 

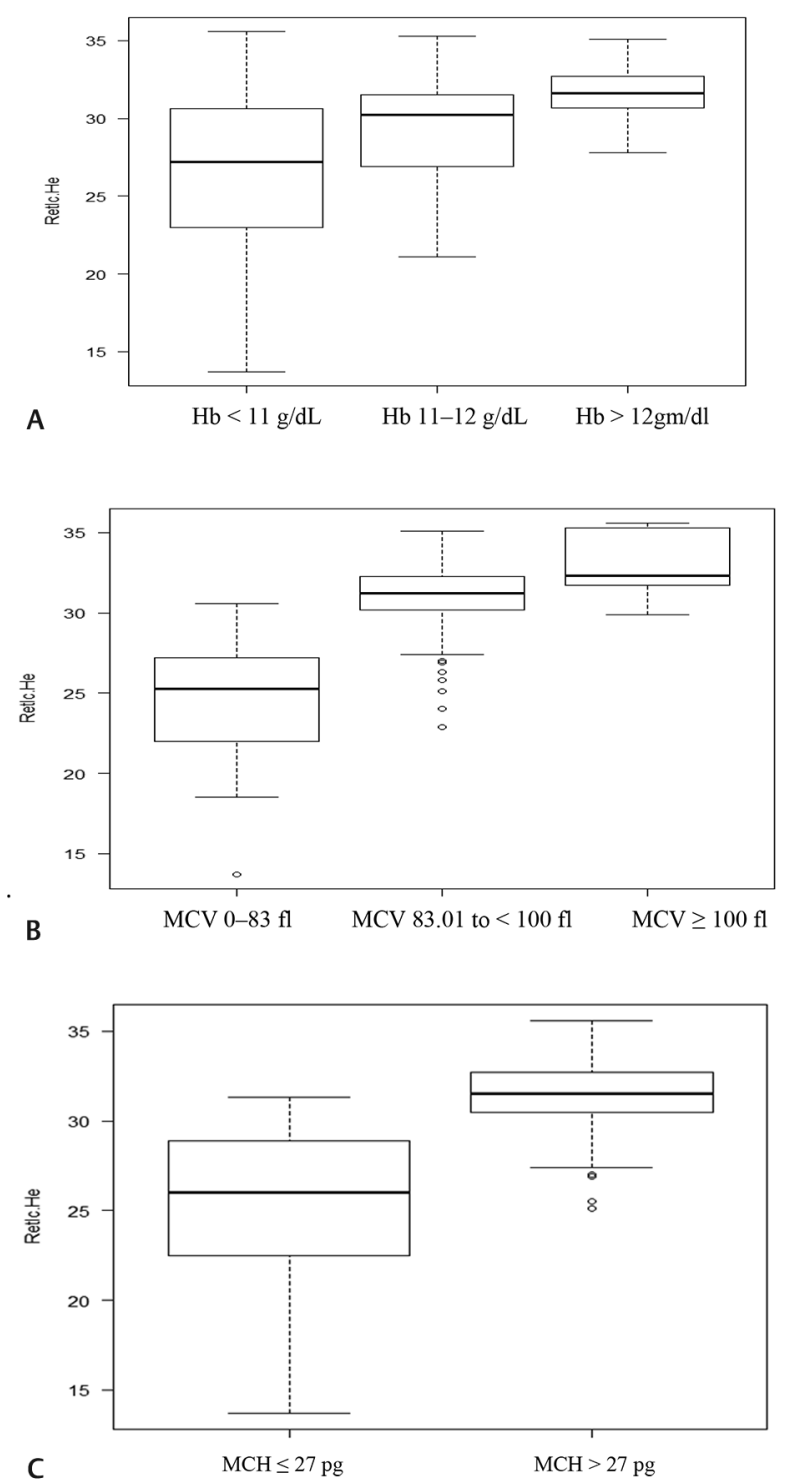

Fig. 1 (a) Box plot analysis of Ret-He in different categories of hemoglobin; (b) Box plot analysis of Ret-He in different categories of MCV; (c) Box plot analysis of Ret-He in different categories of $\mathrm{MCH}$. MCH, mean corpuscular hemoglobin; MCV, mean corpuscular volume; Ret-He, reticulocyte hemoglobin. microcytic hypochromic and normocytic normochromic anemia in pregnant females. Rabindrakumar et al also evaluated hematological indices MCV, MCH, MCHC, and $\mathrm{Hb}$ in predicting early iron deficiency among pregnant females but reticulocyte parameters were not studied. ${ }^{16}$

Another interesting finding observed in the present study was that cutoff of $29.4 \mathrm{ng}$ Ret-He was specific (87\%) for the diagnosis of IDA in pregnant females with positive predictive value of $73 \%$. Previous studies have observed variable cutoffs of Ret-He in IDA ranging from 26 to 30.9 pg. ${ }^{7,11}$ It has also been reported that there is an agreement between the measurements of Ret-He on Sysmex XE 2100 and Bayer ADVIA autoanalyzers and thus eliminating the difference based on instruments. ${ }^{17}$

However, an important limitation of the present study is that females with concomitant thalassemia minor or anemia of chronic disorder (ACD) were not analyzed and therefore the role of reticulocyte parameters in these patients cannot be predicted. Therefore, the authors suggest that further larger studies in pregnant females may be done to assess the role of Ret-He and IRF in diagnosing IDA coexisting with thalassemia minor or ACD.

\section{Conclusion}

Ret-He is a feasible and easily available parameter which may be helpful in differentiating between microcytic hypochromic and normocytic normochromic anemia along with frank and borderline anemia in first trimester pregnant females. This in turn is essential to initiate an early therapy so as to prevent the maternal and fetal complications.

\section{Financial Support}

None.

\section{Conflict of Interest}

None.

Table 4 Serum ferritin and reticulocyte parameters in different categories of mean corpuscular hemoglobin

\begin{tabular}{|l|l|l|l|}
\hline Parameters (Median value) & Category I & Category II & $p$-Value \\
\hline Serum ferritin (ng/mL) & 10.6 & 21.3 & $<0.05$ \\
\hline Ret-He (ng/mL) & 26.3 & 31.6 & $<0.05$ \\
\hline IRF & 12.5 & 10.2 & $<0.05$ \\
\hline RC & 0.06 & 0.067 & 0.6 \\
\hline
\end{tabular}

Abbreviations: IRF, immature reticulocyte fraction; RC, reticulocyte fraction; Ret-He, reticulocyte hemoglobin. 


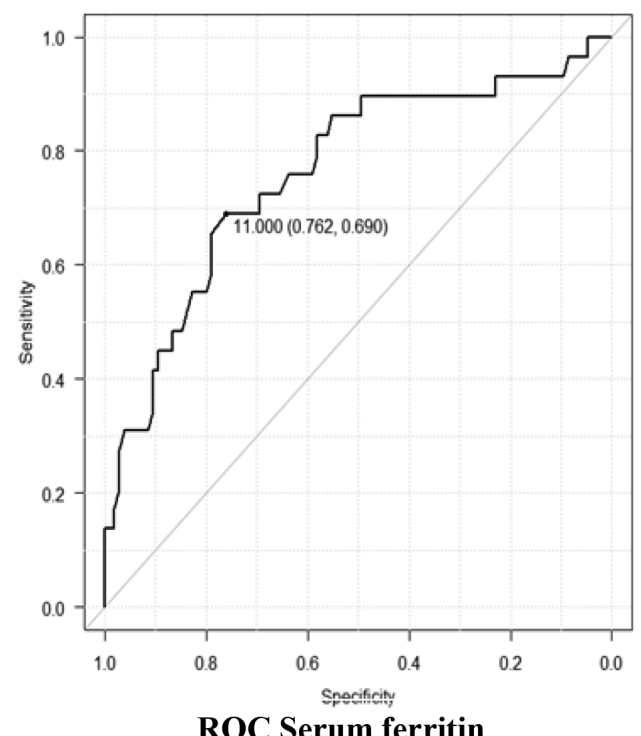

ROC Serum ferritin

AUC 0.76

Cut off $11 \mathrm{ng} / \mathrm{mL}$

95\% CI 0.66-0.87

Sensitivity $76 \%$

a

Specificity $69 \%$



AUC 0.93

Cutoff $27.8 \mathrm{ng} / \mathrm{mL}$ 95\% CI 0.90-0.98

Sensitivity $93 \%$

Specificity $83 \%$

Fig. 2 Receptor operating characteristics curve for (a) serum ferritin and (b) Ret-He at MCV < 83fl and MCH $<27$ pg. MCH, mean corpuscular hemoglobin; MCV, mean corpuscular volume; Ret-He, reticulocyte hemoglobin.

\section{References}

1 WHO, The Global Prevalence of Anaemia in 2011. Geneva: World Health Organization; 2015

2 Kæstel P, Aaby P, Ritz C, Friis H. Markers of iron status are associated with stage of pregnancy and acute-phase response, but not with parity among pregnant women in Guinea-Bissau. Br J Nutr 2015;114(7):1072-1079

3 Costantine MM. Physiologic and pharmacokinetic changes in pregnancy. Front Pharmacol 2014;5:65

$4 \mathrm{WHO} / \mathrm{CDC}$, Assessing the Iron Status of Populations Including Literature Reviews: Report of Joint WHO/CDC Technical Consultation on the Assessment of Iron Status at the Population Level. Geneva: World Health Organisation/Centers for Disease Control and Prevention; 2007

5 Daru J, Allotey J, Peña-Rosas JP, Khan KS. Serum ferritin thresholds for the diagnosis of iron deficiency in pregnancy: a systematic review. Transfus Med 2017;27(3):167-174

6 Malczewska-Lenczowska J, Orysiak J, Szczepańska B, Turowski D, Burkhard-Jagodzińska K, Gajewski J. Reticulocyte and erythrocyte hypochromia markers in detection of iron deficiency in adolescent female athletes. Biol Sport 2017;34(2):111-118

7 Joosten E, Lioen P, Brusselmans C, Indevuyst C, Boeckx N. Is analysis of the reticulocyte haemoglobin equivalent a useful test for the diagnosis of iron deficiency anaemia in geriatric patients? Eur J Intern Med 2013;24(1):63-66

8 Piva E, Brugnara C, Spolaore F, Plebani M. Clinical utility of reticulocyte parameters. Clin Lab Med 2015;35(1):133-163

9 Bobée V, Daliphard S, Schrapp A, Lahary A. Screening of hereditary spherocytosis and pyruvate kinase deficiency by automated blood count using erythrocytic and reticulocytic parameters. Int J Lab Hematol 2018;40(6):697-703
10 Mehta S, Goyal L, Kaushik D, et al. Reticulocyte hemoglobin vis-a-vis immature reticulocyte fraction, as the earliest indicator of response to therapy in iron deficiency anemia. J Assoc Physicians India 2017;65(12):14-17

11 Toki Y, Ikuta K, Kawahara Y, et al. Reticulocyte hemoglobin equivalent as a potential marker for diagnosis of iron deficiency. Int J Hematol 2017;106(1):116-125

12 Viteri FE, Casanueva E, Tolentino MC, Díaz-Francés J, Erazo AB. Antenatal iron supplements consumed daily produce oxidative stress in contrast to weekly supplementation in Mexican non-anemic women. Reprod Toxicol 2012;34(1):125-132

13 Parodi E, Giraudo MT, Ricceri F, Aurucci ML, Mazzone R, Ramenghi U. Absolute reticulocyte count and reticulocyte hemoglobin content as predictors of early response to exclusive oral iron in children with iron deficiency anemia. Anemia 2016;2016(10):1-6

14 Chaipokam J, Nakorn Na T, Rojnuckarin P. Diagnostic accuracy of reticulocyte hemoglobin content in Thai patients with microcytic red cells as a test for iron deficiency anemia. Asian Biomed 2016;10(Suppl 1):S31-S37

15 Tiwari AK, Bhardwaj G, Arora D, et al. Applying newer parameter Ret-He (reticulocyte haemoglobin equivalent) to assess latent iron deficiency (LID) in blood donors-study at a tertiary care hospital in India. Vox Sang 2018;113(7):639-646

16 Rabindrakumar MSK, Pujitha Wickramasinghe V, Gooneratne L, Arambepola C, Senanayake H, Thoradeniya T. The role of haematological indices in predicting early iron deficiency among pregnant women in an urban area of Sri Lanka. BMC Hematol 2018;18:37

17 Brugnara C, Schiller B, Moran J. Reticulocyte hemoglobin equivalent (Ret $\mathrm{He}$ ) and assessment of iron-deficient states. Clin Lab Haematol 2006;28(5):303-308 